\title{
O Diabetes Melito na Fibrose Cística: Uma Comorbidade Cada Vez Mais Freqüente
}

revisõo

\author{
Thais Della ManNa \\ Nuvarte Setian \\ JoAQUim CARLOS RODRIGUES
}

Unidade de Endocrinologia

Pediátrica do Instituto da

Criança do Hospital das Clínicas

da Faculdade de Medicina da

Universidade de São Paulo, SP,

Brasil (TDM, NS); Unidade de

Pneumologia Pediátrica do

Instituto da Criança do Hospital

das Clínicas da Faculdade de

Medicina da Universidade de

São Paulo, SP, Brasil (JCR).

Recebido em 20/1 1/2007

Aceito em 03/12/2007

\author{
RESUMO
}

O diabetes melito (DM) é a principal comorbidade relacionada à fibrose cística (FC) e costuma acontecer após os 15 anos de idade, associando-se ao aumento da morbimortalidade. Sua fisiopatologia inclui destruição do tecido exócrino, insuficiência endócrina e aumento da resistência insulínica, determinando inicialmente a alteração da cinética secretora da insulina, até o comprometimento de sua secreção total. A hiperglicemia pós-prandial é a alteração clínica mais precoce e sintomas do DM podem não ser reconhecidos. A investigação anual deve ser realizada por meio da glicemia de jejum ou de glicemias ao acaso e do teste oral de tolerância à glicose (OGTT). São reconhecidas duas categorias de diabetes relacionadas à FC: o DRFC sem hiperglicemia de jejum (HJ) (glicemia de jejum $<126 \mathrm{mg} / \mathrm{dL}$ e glicemia de 2 horas OGTT > $200 \mathrm{mg} / \mathrm{dL}$ ) e DRFC com HJ (glicemia de jejum $\geq 126 \mathrm{mg} / \mathrm{dL}$ ). $\mathrm{O}$ tratamento inclui o manejo nutricional especializado e a correção da hiperglicemia. A insulinoterapia é recomendada para a categoria DRFC com $\mathrm{HJ}$, não existindo ainda evidências do benefício de drogas secretagogas ou sensibilizantes da ação insulínica. (Arq Bras Endocrinol Metab 2008;52/2:188-197)

Descritores: Diabetes melito; Fibrose cística; Fisiopatologia; Diagnóstico; Tratamento

\section{ABSTRACT}

\section{Cystic Fibrosis-Related Diabetes: A Frequent Co-morbidity.}

Cystic fibrosis-related diabetes (CFRD) is a major co-morbidity generally affecting patients over 15 years old and it is associated with increased morbidity and mortality. The pathophysiology includes exocrine tissue destruction, insulin deficiency and insulin resistance; the carbohydrate metabolism dysfunction begins with an altered kinetic in insulin secretion followed by a progressive insulin deficiency. Postprandial hyperglycemia is the first abnormality seen in CF patients and the classical symptoms of diabetes may not be recognized. The screening strategy proposed is annual random plasma glucose or fasting plasma glucose investigation, as well as the performance the oral glucose tolerance test (OGTT). Two categories of diabetes are related to CF: CFRD without fasting hyperglycemia (fasting glucose $<126 \mathrm{mg} /$ $\mathrm{dL}$ and $2 \mathrm{~h}$ OGTT glucose $>200 \mathrm{mg} / \mathrm{dL}$ ) and CFRD with fasting hyperglycemia (fasting glucose $\geq 126 \mathrm{mg} / \mathrm{dL}$ ). Nutritional management and hyperglycemia control are the CFRD treatment goals. Insulin control is the standard medical therapy for CFRD with fasting hyperglycemia and the benefits of oral insulin secretagogue and sensitizing agents are still controversial. (Arq Bras Endocrinol Metab 2008;52/2:188-197)

Keywords: Cystic Fibrosis-Related Diabetes; Pathophysiology; Diagnosis; Treatment 


\section{INTRODUÇÃO}

A FIBRose cística (FC) é a doença autossômica recessiva letal mais comum entre caucasianos e acontece na freqüência de 1:2.500 a 1:4.000 nascidos vivos $(1,2)$. Sua lesão fisiopatológica primária envolve defeitos na ativação dos canais de cloro $\left(\mathrm{Cl}^{-}\right)$dependentes de adenosina monofosfato cíclico (AMP-cíclico) na superfície apical da membrana celular, afetando a movimentação da água e de outros íons através do epitélio de diversos órgãos, como pulmões, pâncreas, glândulas sudoríparas, fígado, mucosa nasal, glândulas salivares, intestino, ducto deferente, porém em diferentes intensidades (3).

No final da década de 1980, os grupos de Tsui, Riordan e Collins (4-6) isolaram o gene responsável pela FC $(7 \mathrm{q} 31.2)$ e denominaram seu produto genético de "proteína reguladora da condutância transmembrana da fibrose cística" (CFTR), já vislumbrando que suas ações extrapolavam a simples condução do íon cloro.

A CFTR é uma glicoproteína que integra a família dos "transportadores de membrana acoplados ao ATP" (superfamília dos transportadores $A B C$ ), que são capazes de influenciar o funcionamento de outras proteínas de membrana, como receptores acoplados à proteína $\mathrm{G}$, outros canais iônicos $\left(\mathrm{Cl}^{-}, \mathrm{Na}^{+}, \mathrm{K}^{+}\right)$, assim como o canal permutador de $\mathrm{Cl}^{-} / \mathrm{HCO}_{3}^{-}$, interferindo nos processos de fluidificação e acidificação das organelas celulares $(3,7,8)$.

Funcionalmente, as mutações do gene CFTR são classificadas como primárias quando afetam a capacidade de transporte de cloro; secundárias, quando prejudicam o funcionamento de outras proteínas de membrana; terciárias, quando interferem nos processos de acidificação intracelular, no tráfego de glicoproteínas para a superfície celular ou nos mecanismos de endo e exocitoses.

Mais de 900 mutações associadas à FC já foram descritas, sendo a deleção de um resíduo de fenilalanina no códon $508(\Delta \mathrm{F} 508)$ a mais freqüente, correspondendo a aproximadamente $70 \%$ das mutações presentes em populações caucasianas da Europa (1). No Brasil, esta mutação foi descrita na freqüência de $33 \%$ a $52 \%$ em São Paulo (9-11), 49\% no Rio Grande do Sul e 27\% em Santa Catarina (10).
Múltiplos órgãos são afetados pela FC, entretanto, são a insuficiência pancreática e a progressão da doença pulmonar desencadeada pela colonização bacteriana os maiores determinantes de sua morbidade e de seu prognóstico (2). Existe correlação entre as mutações genéticas e a gravidade da insuficiência pancreática e as anormalidades de concentração de cloro no suor, porém é a doença pulmonar que determina a mortalidade na $\mathrm{FC}$ e não existe correlação entre ela e o genótipo. Portanto, outros modificadores genéticos, como também fatores ambientais, devem exercer influência nessa evolução (3).

A taxa de sobrevivência entre os pacientes portadores de FC tem aumentado consideravelmente nas últimas décadas em virtude das recentes condutas na prevenção e no tratamento da doença pulmonar, do estado nutricional e das inúmeras outras manifestações clínicas em crianças e adultos. Nos anos 1960, a expectativa de vida era de quatro anos e hoje a expectativa média de vida para crianças nascidas no ano 2000 é de 40 anos (2).

Com essa maior longevidade, novas complicações têm sido relatadas, entre elas, estão as alterações do metabolismo dos carboidratos na forma de intolerância à glicose até o diabetes melito (DM).

\section{EPIDEMIOLOGIA}

$\mathrm{O}$ DM relacionado à FC (DRFC) é a principal comorbidade em mais de 20 mil portadores de FC notificados no National Cystic Fibrosis Patient Registry que é um registro nacional norte-americano, informatizado, mantido há mais de 30 anos (12).

$\mathrm{O}$ risco de DM na FC é, aproximadamente, 20 vezes maior que na população geral e, freqüentemente, manifesta-se entre 15 e 21 anos de idade. Parece acometer mais indivíduos com comprometimento nutricional e homozigotos para a mutação $\Delta$ F508 (13-15). Não existem marcadores imunológicos ou perfis de HLA (Human Leucocyte Antigens) típicos (16).

A Associação Americana de Diabetes (ADA), desde 1997, classifica o diabetes relacionado à FC na categoria de "outros tipos específicos - doenças do pâncreas exócrino" (17). 


\section{FISIOPATOLOGIA}

A fisiopatologia do DRFC inclui a concorrência dos seguintes mecanismos: presença de anormalidades estruturais do pâncreas exócrino e endócrino; insuficiência funcional do pâncreas endócrino e aumento da resistência periférica à ação da insulina (14,16,18-20).

Existe uma forte associação entre a doença pancreática exócrina e o DRFC. O acometimento exócrino inicia-se pela obstrução dos ductos pancreáticos por mucina, atrofia dos ácinos e substituição por tecido fibrogorduroso, entretanto, no meio de tamanha destruição tecidual, ainda podem ser observadas ilhotas de Langerhans totalmente normais que, ao longo do tempo, passam a apresentar depósitos de amilóide à semelhança do que é visto no $\mathrm{DM}$ do tipo 2 (21-23).

Apesar de 85\% dos portadores de FC apresentarem insuficiência pancreática exócrina, nem todos desenvolverão anormalidades da tolerância à glicose ao longo de suas vidas, provavelmente, um segundo fator etiológico deve determinar o risco individual para o $\operatorname{DRFC}(3,23)$.

A deficiência insulínica é vista, tradicionalmente, como a causa primária do DRFC, porém os estudos não confirmam a existência de uma insuficiência global, mas a presença de uma cinética secretora alterada (18,20,24-27).

Estudos funcionais de avaliação endócrina do pâncreas, utilizando os testes de tolerância à glicose (GTT) intravenoso e oral em portadores de FC, demonstraram um atraso na resposta precoce de insulina, apesar da área sob a curva da resposta insulínica total não diferir da normal. Entre portadores de DRFC com hiperglicemia de jejum também a resposta de insulina total está diminuída $(18,25,26)$.

A resistência à ação da insulina está aumentada em grau discreto em portadores de DRFC e costuma variar em função da gravidade da doença pulmonar, talvez secundariamente ao processo inflamatório subjacente à hipersecreção de citocinas (TNF- $\alpha$, IL-2 e IL-6) ou às drogas empregadas em seu tratamento. A resistência acontece em território hepático, como também é descrita resistência à ação anticatabólica da insulina no metabolismo protéico $(15,26,28,29)$.
A função endócrina do pâncreas na FC apresenta declínio progressivo a partir da puberdade, fase da vida na qual existe reconhecido aumento na resistência insulínica $(15,30,31)$.

\section{QUADRO CLÍ́NICO}

Muitas particularidades da FC influenciam no início e no curso dessa forma de DM, incluindo: desnutrição, infecções agudas e crônicas, gasto energético elevado, malabsorção de nutrientes, trânsito intestinal anormal e disfunção hepatobiliar (16).

A hiperglicemia pós-prandial é a alteração clínica mais precoce. A sintomatologia clássica do diabetes pode não ser reconhecida, uma vez que sintomas como letargia, fraqueza, fadiga, dificuldade na manutenção do peso, desidratação e piora inexplicável da função pulmonar podem ser também manifestações da doença pulmonar e infecções $(16,19)$. A cetoacidose diabética é rara na FC $(14,16,27)$. Complicações diabéticas microvasculares já foram descritas em portadores de FC, mas as macrovasculares ainda não foram reconhecidas $(32,33)$.

Sempre que um paciente com FC apresentar dificuldades na manutenção do peso, a investigação de DM deverá ser realizada.

\section{INVESTIGAÇÃO DIAGNÓSTICA}

O consenso em diabetes da Cystic Fibrosis Foundation, 1998 (16), recomenda pesquisa anual dos distúrbios do metabolismo glicêmico em todo paciente portador de FC por meio da coleta de glicemia ao acaso, principalmente após as refeições, glicemia de jejum e complementação com o teste de tolerância oral à glicose (OGTT) em alguns casos, considerando-se a baixa sensibilidade e especificidade da glicemia de jejum (34). A triagem sistemática para o DRFC é recomendada principalmente após os 14 anos de idade (35).

Conforme esse consenso, a classificação das categorias de tolerância à glicose será feita com base na presença ou na ausência da glicemia de jejum diabética $(\geq$ $126 \mathrm{mg} / \mathrm{dL}$ ), confirmada em pelo menos duas ocasiões diferentes, assim como na glicemia de duas horas do OGTT $(14,16)$ (Tabela 1). 
Tabela 1. Classificação das categorias de tolerância à glicose na FC, segundo as diretrizes do consenso em diabetes da Fundação de Fibrose Cística (1998).

\begin{tabular}{lcc}
\hline Categorias & Glicemia de jejum $(\mathbf{m g} / \mathbf{d L})$ & Glicemia de $2 \mathrm{~h}$ GTT oral $(\mathbf{m g} / \mathbf{d L})$ \\
TG Normal & $<126$ & $<140$ \\
IGT & $<126$ & $140-200$ \\
DRFC sem HJ & $<126$ & $>200$ \\
DRFC com HJ & $\geq 126$ & GTT oral não é necessário \\
\hline
\end{tabular}

TG = tolerância à glicose; IGT = tolerância alterada ò glicose; DRFC = diabetes relacionado à fibrose cística;

$\mathrm{HJ}=$ hiperglicemia de jejum.

Conseqüentemente, foram reconhecidas duas categorias de DM na FC: o DM relacionado à FC sem hiperglicemia de jejum (DRFC sem HJ) e o DM relacionado à FC com hiperglicemia de jejum (DRFC com HJ). A hiperglicemia de jejum pode estar presente o tempo todo (DRFC crônica) ou somente em situações de descompensação aguda (DRFC intermitente) (16).

A prevalência de DRFC está por volta de $5 \%$ a $6 \%$ em clínicas especializadas da América do Norte e da Europa; na Dinamarca, onde se realiza anualmente o OGTT em portadores de FC, relata-se diabetes em cerca de $50 \%$ dos pacientes com mais de 30 anos $(12,13,36,37)$.

O centro de FC da Universidade de Minnesota realiza OGTT, anualmente, em todo paciente maior de cinco anos de idade e estima-se que $35 \% \pm 2 \%$ dos pacientes apresentem tolerância normal à glicose; $37 \% \pm$ $2 \%$ têm intolerância à glicose (IGT); $17 \% \pm 2 \%$ têm DRFC sem HJ e $11 \%$ têm DRFC com HJ. A proporção de pacientes portadores de FC que desenvolvem DRFC aumentou com a idade, sendo que a prevalência de DRFC, incluindo casos com e sem HJ, foi de $9 \%, 26 \%$, $35 \%$ e $43 \%$ em pacientes com idades entre 5 e 9 anos, 10 e 19 anos, 20 e 30 anos e maiores de 30 anos, respectivamente (28). Entretanto, quando se analisa o registro de pacientes da Cystic Fibrosis Foundation pela freqüência de pacientes em insulinoterapia, a prevalência de DM insulino-dependente em pacientes menores de 10 anos e adultos maiores de 18 anos, nos Estados Unidos, essa prevalência cai para $1 \%$ e $8 \%$, respectivamente (12).

No Instituto da Criança do Hospital das Clínicas da Faculdade de Medicina da Universidade de São Paulo, pacientes com distúrbios glicêmicos relacionados à FC correspondem à segunda população de maior prevalência acompanhada no Ambulatório de Diabetes. Entre 2000 e 2004, realizando o OGTT anualmente em 56 portadores de FC, verifica-se falta de concordância entre as respostas glicêmicas sucessivas, havendo reclassificação diagnóstica em 15 casos, porém não necessariamente no sentido do maior comprometimento da tolerância à glicose, uma vez que quatro pacientes com IGT e dois com DRFC sem HJ foram evolutivamente reclassificados como normais, além de um paciente com DRFC sem HJ tornar-se IGT (26) (Figura 1).

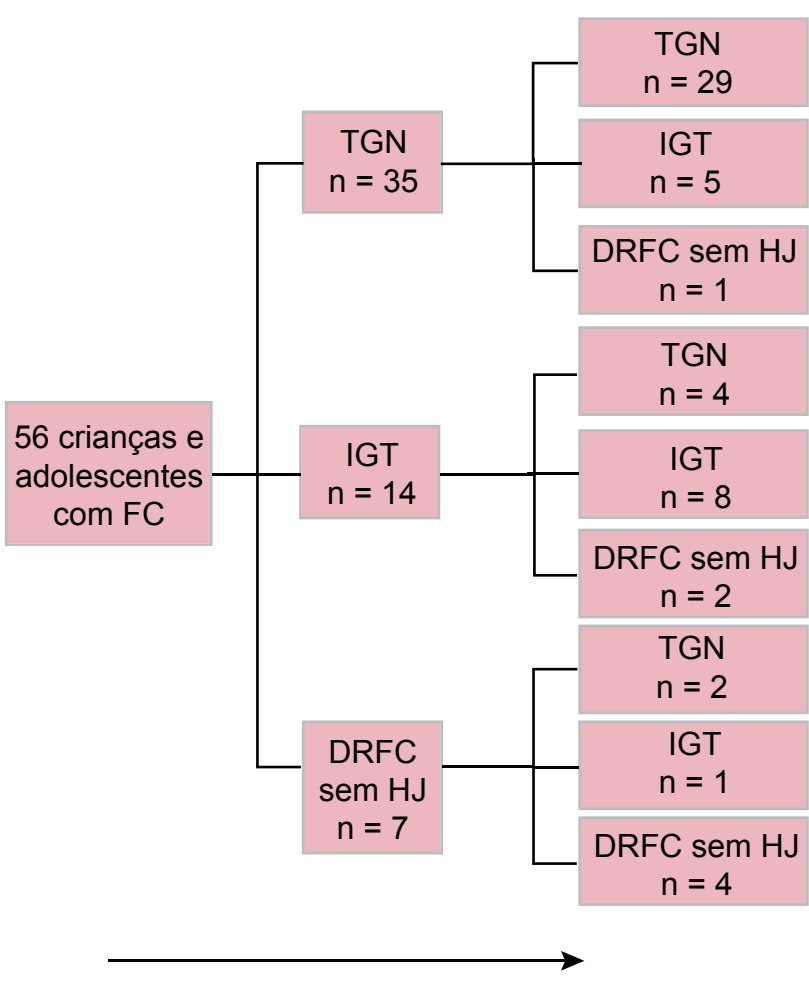

Julho de 200 a dezembro de 2004

TGN = tolerância à glicose normal; IGT = intolerância à glicose; DRFC sem HJ = diabetes relacionado à fibrose cística sem hiperglicemia de jejum.

Figura 1. Classificação das categorias de tolerância à glicose pelo OGT no início e no final do período entre julho/2000 e dezembro/2004. 
Esta falta de concordância entre as respostas glicêmicas de testes sucessivos, assim como ausência de glicosúria, normalidade dos perfis de glicemias capilares e dos níveis de hemoglobina glicada em pacientes com distúrbios glicêmicos diagnosticados pelo OGTT, trouxe motivação a realizar um estudo entre julho de 2004 e agosto de 2005, comparando as respostas de glicose, insulina, proinsulina, peptídeo-C e glucagon obtidas no OGTT com aquelas obtidas num teste de tolerância à dieta mista (TTDM) isocalórica e isosmolar (26).

A partir de 2004, também passamos a adotar em nosso serviço os critérios de diagnóstico e classificação do DM sugeridos pela American Diabetes Association, 2004 (38), definindo como pré-diabéticos (PD) pacientes com glicemia de jejum entre 100 e $125 \mathrm{mg} / \mathrm{dL}$, como também aqueles com glicemia de duas horas do OGTT entre 140 e $200 \mathrm{mg} / \mathrm{dL}$.

$\mathrm{O}$ estímulo da dieta mista foi manipulado com maior eficiência pelo paciente portador de FC, por apresentar uma curva glicêmica com tendência à queda já a partir dos 60 minutos e retorno aos níveis de jejum aos 180 minutos. Somente a esse estímulo estiveram associadas respostas precoces de insulina e peptídeo-C (30 minutos) (Figura 2). Essa resposta precoce, provavelmente, reflete a capacidade da ilhota em responder a estímulos diferentes da glicose, como os aminoácidos e gorduras da dieta mista ou por estímulos hormonais gastrintestinais (incretinas) gerados por ela.

Por outro lado, o grupo portador de DRFC com HJ apresentou atraso do pico de resposta de insulina e peptídeo-C para o tempo 90 minutos, sem, contudo, apresentar diferenças nas áreas sob a curva (AUC) de insulina e peptídeo-C em relação aos demais grupos, apesar da AUC de glicose significativamente superior (Figura 2).

Ao grupo portador de DRFC sem HJ associaramse respostas mais tardias e elevadas de insulina, peptídeo-C e proinsulina. A AUC de proinsulina também foi significativamente mais elevada no grupo DRFC sem HJ, demonstrando a participação da resistência insulínica na fisiopatologia dessa forma de distúrbio glicêmico (Figura 2).

São fortes as evidências indicando que a disfunção primária associada aos distúrbios glicêmicos na FC seja a alteração da cinética secretora de insulina $(20,30,39,40)$ e, uma vez que a uma rápida elevação da glicemia pósprandial segue-se uma resposta insulínica atrasada, mas prolongada, esses pacientes costumam apresentar sintomas tanto de hiper quanto de hipoglicemia (41).

\section{TRATAMENTO}

A condução do tratamento do paciente com $\mathrm{FC}$ requer abordagem multiprofissional que inclui pneumologista, gastroenterologista, nutricionista, fisioterapeuta, enfermeira, psicólogo, endocrinologista, cardiologista e cirurgião de tórax, quando da indicação do transplante de pulmão. Além disso, esse paciente já faz uso de inúmeros medicamentos, como: enzimas pancreáticas, vitaminas lipossolúveis, triglicérides de cadeia média, suplementos alimentares hipercalóricos, ácido ursodesoxicólico, antiácidos, procinéticos, inalações com dornase alfa e aminoglicosídeos, como tobramicina ou gentamicina, corticosteróides orais ou nasais, drogas broncodilatadoras e antibióticos orais e parenterais com muita freqüência.

A orientação do tratamento do DRFC difere daquele do DM tipo 1 e do DM tipo 2 basicamente porque, enquanto a morbimortalidade desses últimos está associada à nefropatia e doença cardiovascular, no DRFC ela está relacionada à desnutrição e à doença pulmonar.

Os objetivos são a otimização do crescimento e do desenvolvimento, com manutenção do índice de massa corpórea (IMC) acima do percentil 25 e oferta calórica entre $10 \%$ e $40 \%$ acima das necessidades diárias recomendadas para sexo e idade. Indicadores de agravo nutricional são a queda da velocidade de crescimento e a falta de ganho de peso por seis meses consecutivos (19). As recomendações dietéticas específicas para a FC devem prevalecer sobre aquelas habitualmente empregadas para outras formas de diabetes (16).

Portanto, o enfoque principal no tratamento do DRFC é o manejo nutricional e o tratamento da hiperglicemia inicialmente no período pós-prandial e, evolutivamente, também no estado de jejum.

O cálculo calórico total será o somatório dos $110 \%$ aos $140 \%$ das necessidades diárias recomendadas para sexo e idade com o gasto energético do paciente, e deverá ser distribuído nas diversas refeições do dia, incluindo uma suplementação alimentar noturna por sonda nasogástrica ou gastrostomia em alguns pacientes $(19,42)$.

A dieta hipercalórica deverá ser composta por 35\% a $40 \%$ de gorduras, $15 \%$ a $20 \%$ de proteínas e $40 \%$ a $50 \%$ de carboidratos de cadeia complexa, apesar de não haver restrição para açúcares simples (35).

Fracionando-se as calorias calculadas nas porcentagens recomendadas de carboidratos, proteínas e gordu- 
Painel A
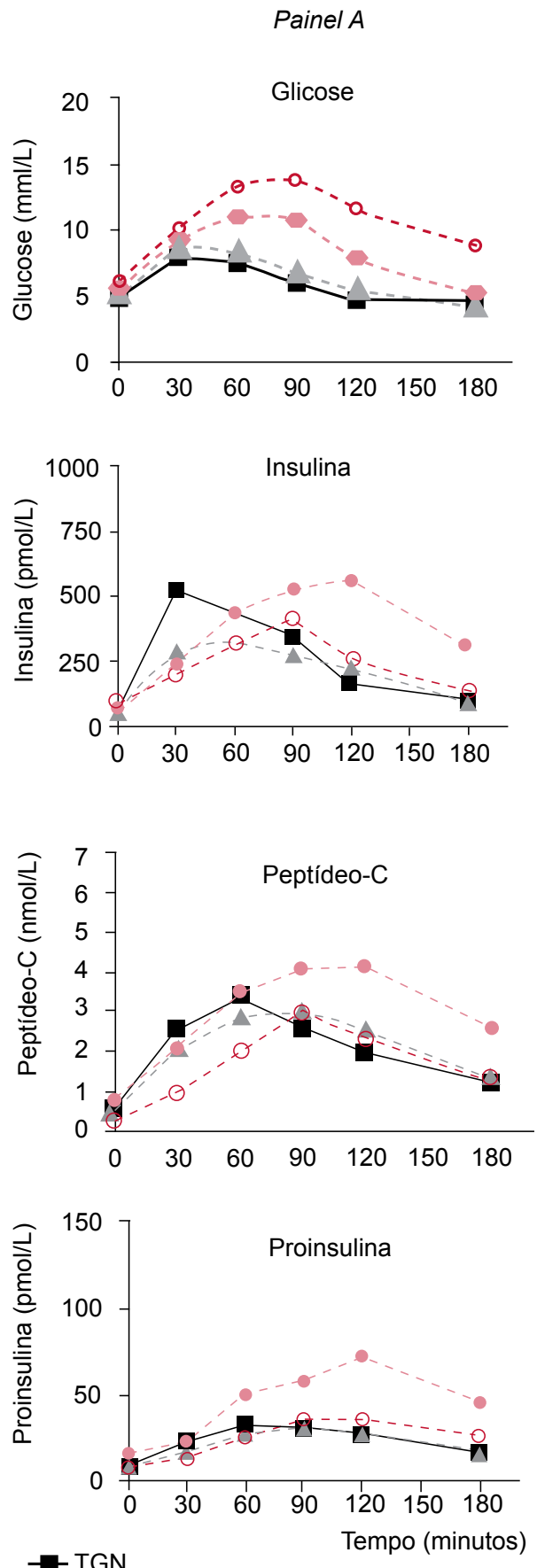

- TGN

- Pré-diabetes

- DRFC s/ HJ

$-\odot-D R F C \mathrm{c} / \mathrm{HJ}$
Painel B

AUC Glicose
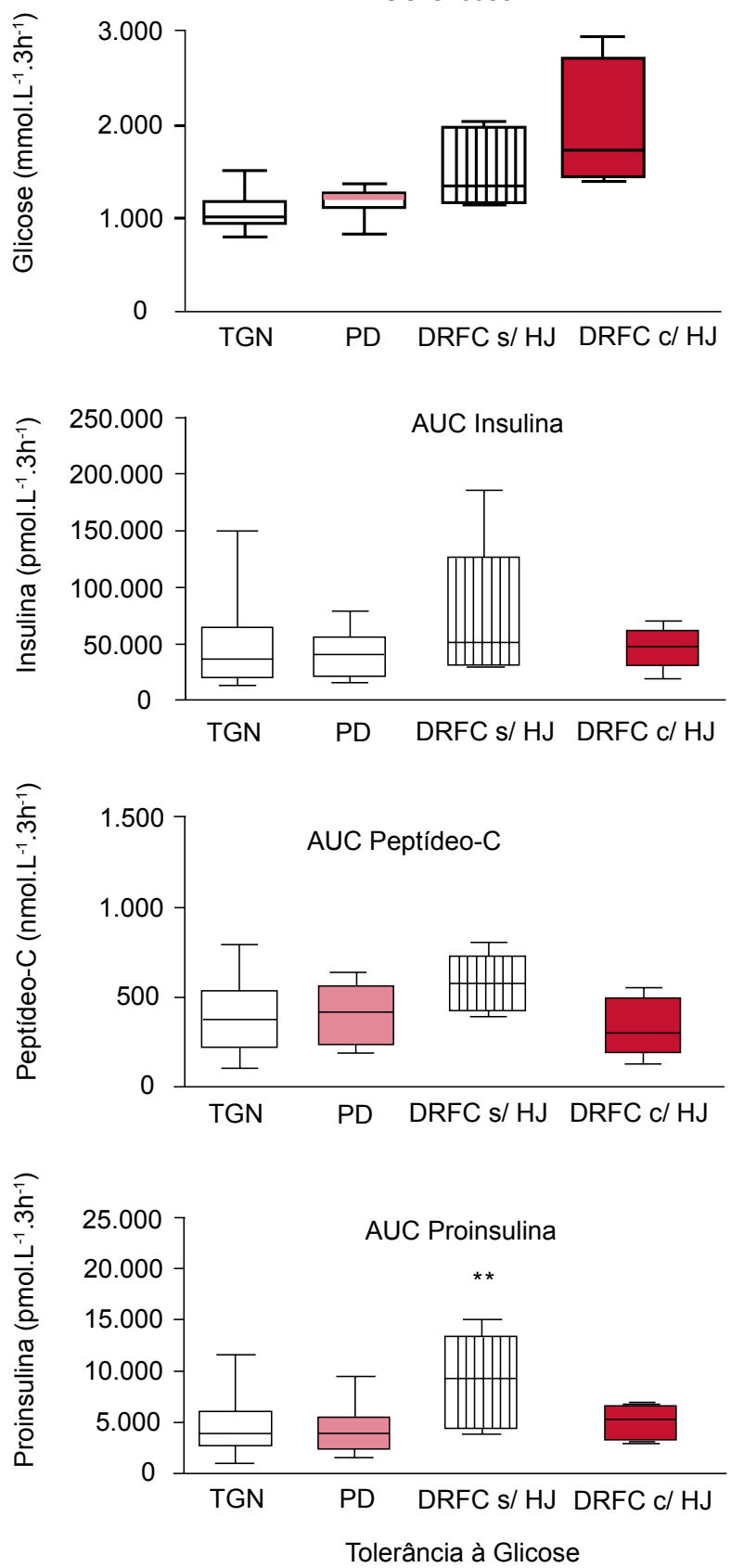

TGN = tolerância à glicose normal; PD = pré-diabetes; DRFC s/ HJ = diabetes relacionado à fibrose cística sem hiperglicemia de jejum; DRFC c/ HJ = diabetes relacionado à fibrose cística com hiperglicemia de jejum. (ANOVA: ${ }^{* *} p<0,01$ )

Figura 2. Médias das respostas hormonais e de glicose obtidas durante o teste de tolerância à dieta mista (Painel A) na população do estudo agrupada conforme o diagnóstico de tolerância à glicose, segundo os critérios da ADA (2004) e representação das áreas sob a curva (AUC) dessas respostas (Painel B). 
ras, pode-se combinar a quantidade de carboidratos, transformada em gramas, com a dose de insulina de ação ultra-rápida por intermédio das técnicas de substituição e contagem de carboidratos, na proporção 1 UI de insulina para cada 15 gramas de carboidrato ou em proporções mais individualizadas, adicionando-se algumas vezes uma dose suplementar para correção da hiperglicemia pré-prandial $(19,35)$.

Tal estratégia só será possível por meio da automonitorização glicêmica freqüente realizada por punção da ponta de dedo ou por intermédio do sistema de monitorização contínua de glicose que parece apresentar boa sensibilidade para o diagnóstico de hiperglicemias em portadores de FC $(43,44)$. A Cystic Fibrosis Foundation recomenda que as metas glicêmicas sejam diferenciadas segundo a faixa etária do paciente (45) (Tabela 2), e as diretrizes do Reino Unido recomendam um controle glicêmico bastante rigoroso, porém diferenciam as metas para pacientes em risco de hipoglicemia e para aqueles em fases mais terminais (46) (Tabela 3).

O tratamento da hiperglicemia será direcionado primeiramente ao controle pós-prandial por meio da introdução da insulinoterapia relacionada às refeições, de preferência com análogos de ação rápida (lispro ou aspart). Evolutivamente, haverá necessidade da introdução da insulinoterapia basal para controle da hiperglicemia de jejum com insulina humana de ação intermediária ou análogos de ação lenta (glargina ou detemir) antes de dormir. Pode ainda ser necessária a introdução de insulinoterapia para controle da hiperglicemia associada à dieta enteral noturna, sendo interessante a administração de insulina humana regular uma hora antes do início da infusão enteral.

Apesar das poucas evidências clínicas, existem pacientes que são controlados com antidiabéticos orais secretagogos de insulina, como glinidas (repaglinida) (47) e sulfoniluréias (glibenclamida) $(48,49)$, enquanto a relação risco-benefício é ainda incerta para a utilização de agentes sensibilizantes da ação insulínica, como a metformina e as tiazolidinedionas (49). A acarbose, um inibidor da enzima alfa-glicosidase, apesar de reduzir a glicemia pós-prandial, causa diarréia, anorexia e desconforto abdominal, não sendo indicada para pacientes desnutridos $(16,41)$.

Não existem ainda diretrizes para o tratamento do DRFC sem HJ uma vez que o risco para complicações microvasculares ou agravos nutricionais não parece significativo. A triagem glicêmica deverá ser intensificada nos períodos de agudização pulmonar quando o aumento da resistência insulínica poderá precipitar a hiperglicemia. A insulinoterapia deverá ser considerada nesses episódios, assim como em situações de perda de peso e retardo do crescimento e desenvolvimento puberal. Ainda não existem evidências a favor do tratamento dessa condição com antidiabéticos orais, que devem ser considerados somente em estudos controlados (16).

Tabela 2. Metas glicêmicas recomendadas pela Cystic Fibrosis Foundation (2002).

\begin{tabular}{lcc}
\hline Horários & $\mathbf{5}-\mathbf{1 2}$ anos & $>12$ anos \\
\hline Pré-prandial $(\mathrm{mg} / \mathrm{dL})$ & $80-180$ & $80-120$ \\
Pós-prandial $\left(1 \frac{1}{2}\right.$ a $2 \mathrm{~h}$ após) $(\mathrm{mg} / \mathrm{dL})$ & $<150$ & $<150$ \\
Antes de dormir $(\mathrm{mg} / \mathrm{dL})$ & $100-160$ & $100-140$ \\
\hline
\end{tabular}

Tabela 3. Metas glicêmicas segundo UK Cystic Fibrosis Trust (2004).

\begin{tabular}{lccc}
\hline Horários & Ótimo & Risco de hipoglicemia & Cuidados paliativos \\
\hline Pré-prandial (mg/dL) & $70-110$ & $70-126$ & $<180$ \\
Pós-prandial (1 $1 / 2$ a 2 h após) $(\mathrm{mg} / \mathrm{dL})$ & $70-126$ & $70-180$ & $<180$ \\
HbAlc $(\%)$ & $<7,0$ & $<8,0$ & Irrelevante \\
\hline
\end{tabular}


Um número crescente de mulheres portadoras de FC tem chegado à gestação $\mathrm{e}, \mathrm{com}$ alguma freqüência, $\mathrm{o}$ diabetes é diagnosticado somente nessa fase. Sabe-se que algumas portadoras de DRFC podem apresentar rápida deterioração pulmonar nos anos subseqüentes à gestação, portanto, um controle metabólico ótimo é recomendado antes da concepção, assim como um aumento da oferta nutricional. Seria aconselhável a realização do OGTT quando se planeja engravidar e repeti-lo entre a $20^{\mathrm{a}}$ e $24^{\mathrm{a}}$ semanas e entre a $30^{\mathrm{a}}$ e $34^{\mathrm{a}}$ semanas (16).

\section{PROGNÓSTICO}

O surgimento do DM parece acrescentar maior gravidade à doença fibrocística. A taxa de mortalidade é seis vezes maior entre os portadores de DRFC com HJ e, destes, menos de $25 \%$ sobrevivem aos 30 anos de idade (50); dos cinco pacientes portadores de DRFC com HJ incluídos em nosso estudo (26), três foram a óbito nos últimos dois anos. Existe ainda uma forte associação com o agravamento da função pulmonar e do estado nutricional (36).

As complicações diabéticas microvasculares acontecem no DRFC com HJ, porém a prevalência de retinopatia (16\%) e microalbuminúria (14\%), após dez anos de doença é inferior àquela do DM tipo 1 e tipo 2 , relacionando-se também com o tempo de diabetes e grau de controle metabólico. Neuropatia periférica e gastroparesia são descritas em uma freqüência semelhante às outras formas de diabetes. Sugere-se triagem anual para as complicações microvasculares após cinco anos do diagnóstico do DRFC com HJ (33). O risco para as complicações macrovasculares parece não ser significativo, até o momento, e níveis de colesterol e triglicérides persistem normais na população adulta portadora de $\operatorname{DRFC~(16).~}$

Com o tempo, a maioria dos portadores de DRFC sem HJ evoluirá para a hiperglicemia de jejum, com aumento de $45 \% \mathrm{em}$ cinco anos para $60 \% \mathrm{em} \mathrm{dez}$ anos de acompanhamento (33).

Portadores de FC com IGT apresentam risco mais alto para o DM que aqueles com tolerância normal (27), mas é ainda controverso, se o estado pré-diabético apresenta relação com o declínio da função pulmonar e sobrevida dos pacientes $(16,20,50)$, assim como ainda é especulativo se a intervenção precoce com insulina ou outra droga antidiabética possa interferir nessa evolução $(36,48)$.

\section{CONCLUSÃo}

Os portadores de FC apresentam risco aumentado para anormalidades do metabolismo dos carboidratos, tendo como base fisiopatológica a falência da célula betapancreática, associada à resistência insulínica, em progressão mais rápida do que aquela vista em pacientes com DM tipo 2. As diretrizes vigentes para o tratamento dos distúrbios glicêmicos na FC não preconizam intervenção terapêutica em outro estado que não seja o DRFC com $\mathrm{HJ}$ por ainda não existirem evidências em seu favor.

Diante dos recentes avanços da terapêutica do diabetes melito, são necessários estudos que avaliem a eficácia do tratamento precoce das alterações glicêmicas do paciente com FC tão logo sejam diagnosticadas, em vez de se continuar assistindo a falência progressiva da secreção insulínica para, então, introduzir insulinoterapia periférica.

\section{REFERÊNCIAS}

1. The Cystic Fibrosis Genotype-Phenotype Consortium. Correlation between genotype and phenotype in patients with cystic fibrosis. N Engl J Med. 1993;329:1308-13.

2. Garagorri JM, Rodríguez G, Ros L, Sánchez A. Early detection of impaired glucose tolerance in patients with cystic fibrosis and predisposition factors. J Pediatr Endocrinol Metab. 2001;14:53-60.

3. Welsh MJ, Ramsey BW, Accurso F, Cutting GR. Cystic Fibrosis. In: Scriver CR, Beaudet AL, Sly WS, Valle D, editors. The metabolic and molecular bases of inherited disease, volume III. New York: McGraw-Hill; 2002. p. 5121-87.

4. Kerem B, Rommens JM, Buchanan JA, Markiewicz D, Cox TK, Chakravarti $A$, et al. Identification of the cystic fibrosis gene: genetic analysis. Science. 1989;245:1073-80.

5. Riordan JR, Rommens JM, Kerem B, Alon N, Rozmahel R, Grzelczar $Z$, et al. Identification of the cystic fibrosis gene: cloning and characterization of the complementary DNA. Science. 1989;245:1066-73.

6. Rommens JM, lannuzzi MC, Kerem B, Drumm ML, Melmer G, Dean $M$, et al. Identification of the cystic fibrosis gene: chromosome walking and jumping. Science. 1989;245:1059-65.

7. Schwiebert EM, Benos DJ, Egan ME, Stutts MJ, Guggino WB. CFTR is a conductive regulator as well as a chloride channel. Physiol Rev. 1999;78(1):S145-66.

8. Dean M, Rzhetsky A, Allikmets R. The human ATP-binding cassette $(A B C)$ transporter superfamily. Genome Res. 2001;11: 1156-66.

9. Ribeiro JC, Ribeiro MAGO, Ribeiro AF. Controvérsias na fibrose cística - do pediatra ao especialista. J Pediatr (Rio J). 2002;78(Supl.2):S171-86.

10. Raskin S, Phillips JA 3rd, Krishnamani MR, Vnencak-Jones $C$ Parker RA, Rozov T, et al. DNA analysis of cystic fibrosis in Brazil by direct PCR amplification from Guthrie cards. Am J Med Gen. 1993;46:665-9. 
11. Martins CSB, Ribeiro AF, Costa FF. Frequency of the cystic fibrosis DF508 mutation In a population from São Paulo State, Brazil. Braz J Med Biol Res. 1993;26:1037-40.

12. Cystic Fibrosis Foundation Patient Registry. Annual data base report. Bethesda, MD: Cystic Fibrosis Foundation; 1997.

13. Rosenecker J, Eichler I, Kuhn L, Harms HK, von der Hardt H. Genetic determination of diabetes mellitus in patients with cystic fibrosis. J Pediatr. 1995;127:441-3.

14. Hardin DS, Moran A. Diabetes mellitus in cystic fibrosis. Endocrinol \& Metab Clin N Am. 1999;28:787-801.

15. Lanng S, Thorsteinsson B, Lund-Andersen C, Nerup J, Schioz PO, Koch C. Diabetes mellitus in Danish cystic fibrosis patients: prevalence and late diabetic complications. Acta Paediatr. [Scand] 1994;83:72-7.

16. Moran A, Hardin D, Rodman D, et al. Diagnosis, screening and management of cystic fibrosis related diabetes mellitus: aconsensus conference report. Diabetes Res Clin Pract. 1999; 45:61-73.

17. American Diabetes Association. Clinical Practice Recommendations 1999. Diabetes Care. 1999;22(Suppl 1):S7.

18. Moran A, Diem P, Klein DJ, Levitt MD, Robertson P. Pancreatic endocrine function in cystic fibrosis. J Pediatr. 1991;118:715-23.

19. Moran A. Endocrine complications of cystic fibrosis. Adolesc Med. 2002;13(1):145-59.

20. Brennan AL, Geddes DM, Gyi KM, Baker EH. Clinical importance of cystic fibrosis-related diabetes. J Cystic Fibrosis. 2004;3(4):209-22.

21. Iannucci A, Mukai K, Johnson D, Burke B. Endocrine pancreas in cystic fibrosis: an immunohistochemical study. Hum Pathol. 1984;15(3):278-84.

22. Couce M, O'Brien TD, Moran A, Roche PC, Butler PC. Diabetes mellitus in cystic fibrosis is characterized by islet amyloidosis. J Clin Endocrinol Metab. 1996;81:1267-72.

23. Lohr M, Goertchem P, Nizze $\mathrm{H}$, et al. Cystic fibrosis associated islet changes may provide a basis for diabetes. Virchows Arch. 1989;414:179-85.

24. Austin A, Kalhan SC, Orenstein D, Nixon P, Arslanian S. Roles of insulin resistance and beta-cell dysfunction in the pathogenesis of glucose intolerance in cystic fibrosis. J Clin Endocrinol Metab. 1994;79:80-5.

25. DeSchepper J, Hachimi-Idrissi S, Smitz J, Dab I, Loeb H. Firstphase insulin release in adult cystic fibrosis patients: correlation with clinical and biological parameters. Horm Res. 1992;38:260-3.

26. Della Manna T. Avaliação do comprometimento endócrino do pâncreas em crianças e adolescentes portadores de fibrose cística. [Tese Doutorado]. São Paulo: Faculdade de Medicina da Universidade de São Paulo; 2006. Disponível em: http://www. teses.usp.br/teses/disponíveis/5/5141/tde-11042007-112815/

27. Lanng S, Hansen A, Thorsteinsson B, Nerup J, Koch C. Glucose tolerance in cystic fibrosis: a five year prospective study. BMJ. 1995;311:655-9.

28. Moran A, Doherty L, Wang X, Thomas W. Abnormal glucose metabolism in cystic fibrosis. J Pediatr. 1998;133:10-7.

29. Hardin DS, LeBlanc A, Lukenbaugh S, Seilheimer DK. Insulin resistance is associated with decreased clinical status in cystic fibrosis. J Pediatr. 1997;6:948-56.

30. Ball GD, Huang TT, Gower BA, Cruz ML, Shaibi GQ, Weigensberg $\mathrm{MJ}$, et al. Longitudinal changes in insulin sensitivity, in- sulin secretion, and beta-cell function during puberty. J Pediatr. 2006;148:16-22.

31. Hannon TS, Janosky J, Arslanian SA. Longitudinal study of physiological insulin resistance and metabolic changes of puberty. Pediatr Res. 2006;60:759-63.

32. Dobson L, Stride A, Bingham C, Elworthy S, Sheldon CD, Hattersley AT. Microalbuminuria as a screening tool in cystic fibrosis related diabetes. Pediatr Pulmonol. 2005;39:103-7.

33. Schwarzenberg SJ, Thomas W, Olsen TW, Grover T, Walk D, Milla $\mathrm{C}$, et al. Microvascular complications in cystic fibrosisrelated diabetes. Diabetes Care. 2007;30(5):1056-61.

34. National Diabetes Data Group. Classification and diagnosis of diabetes mellitus and other categories of glucose intolerance. Diabetes. 1979;28:1039-57.

35. Costa M, Potvin S, Berthiaume Y, Gauthier L, Jeanneret A, Lavoie $A$, et al. Diabetes: a major co-morbidity of cystic fibrosis. Diabetes Metab. 2005;31:221-32.

36. Koch C, Cuppens H, Rainisio M, Madessani U, Harms HK, Hodson ME, et al. European Epidemiologic Registry of Cystic Fibrosis (ERCF): Comparison of major disease manifestations between patients with different classes of mutations. Pediatr Pulmonol. 2001;31:1-12.

37. Marshall BC, Butler SM, Stoddard M, Moran AM, Liou TG, Morgan WJ. Epidemiology of cystic fibrosis-related diabetes. J Pediatr. 2005;146:681-7.

38. American Diabetes Association: Diagnosis and classification of diabetes mellitus (Positional Statement). Diabetes Care. 2004;27(Suppl 1):S5-10.

39. Yung B, Noormohamed FH, Kemp M, Hooper J, Lant AF, Hodson ME. Cystic fibrosis-related diabetes: the role of peripheral insulin resistance and beta-cell dysfunction. Diabet Med. 2002;19(3):221-6

40. Tofé S, Moreno JC, Máiz L, Alonso M, Escobar H, Barrio R. Insulin-secretion abnormalities and clinical deterioration related to impaired glucose tolerance in cystic fibrosis. Eur J Endocrinol. 2005;152:241-7.

41. Kentrup $H$, Bongers $H$, Spengler M, Kusenbach G, Skopnik $H$. Efficacy and safety of acarbose in patients with cystic fibrosis and impaired glucose tolerance. Eur J Pediatr. 1999;158(6):455-9.

42. Hardin DS, Cohen R, Clark J, Kriz D, Spears S. Medical \& Nutritional Management of CFRD. Sixteenth Annual North American CF Conference of Cystic Fibrosis Foundation, New Orleans, October 3, 2002.

43. Dobson L, Sheldon CD, Hattersley AT. Conventional measures underestimate glycemia in cystic fibrosis patients. Diabet Med. 2004;21(7):691-6.

44. Jefferies C, Solomon M, Perlman K, Sweezey N, Daneman D. Continuous glucose monitoring in adolescents with cystic fibrosis. J Pediatr. 2005;147:396-8.

45. Hardin DS, Brunzell C, Schissel K, Schindler T, Moran A. Managing Cystic fibrosis-related diabetes (CFRD). An instruction guide for patients and families. 3rd ed. Cystic Fibrosis Foundation. 2002. Bethesda, USA. Cystic Fibrosis Foundation. Disponível em: http://www.cff.org/UploadedFiles/LivingWithCF/ StayingHealthy/Diet/Diabetes/CFRDManual.pdf

46. UK cystic fibrosis trust diabetes working group. Management of cystic fibrosis related diabetes mellitus. Cystic fibrosis Trust. 2004. Bromley, Kent UK, Cystic fibrosis Trust. Disponível em: http://www.cftrust.org.uk/aboutcf/publications/consensusdoc/ diabetes.pdf 
47. Moran A, Phillips J, Milla C. Insulin and glucose excursions following premeal insulin lispro or repaglinide in cystic fibrosis-related diabetes. Diabetes Care. 2001;24:1706-10.

48. Rosenecker J, Eichler I, Barmeier H, von der HH. Diabetes mellitus and cystic fibrosis: comparison of clinical parameters in patients treated with insulin versus oral glucose-lowering agents. Pediatr Pulmonol. 2001;32:351-5.

49. Onady GM, Langdon LJ. Insulin versus oral agents in the management of cystic fibrosis related diabetes: a case based study. BMC Endocrinol Dis. 2006;6:4. Disponível em: http://www. biomedcentral.com/1472-6823/6/4
50. Lanng S, Thorsteinsson B, Roder ME, Nerup J, Koch C. Insulin sensitivity and insulin clearance in cystic fibrosis patients with normal and diabetic glucose tolerance. Clin Endocrinol. 1994;41:217-23.

Endereço para correspondência:

Thais Della Manna

Av. Pedroso de Moraes, 427

05419-000 São Paulo, SP

E-mail: thaisdm@icr.hcnet.usp.br 